\title{
Novidades taxonômicas para Dilleniaceae brasileiras ${ }^{1}$
}

\author{
Taxonomic novelties to Brazilian Dilleniaceae
}

\author{
Claudio Nicoletti de Fraga ${ }^{2}$ \& João Renato Stehmann ${ }^{3}$
}

\begin{abstract}
Resumo
São apresentadas novidades taxonômicas para Dilleniaceae da flora brasileira provenientes da revisão de dados bibliográficos e da revisão de herbários. Uma nova espécie, Davilla lanosa Fraga \& Stehmann, é descrita, ilustrada e comentada acerca dos caracteres diagnósticos e das afinidades com outras espécies do gênero, sendo conhecida de duas localidades em Rondônia e uma ao sul do Amazonas, Brasil. É também estabelecida uma nova combinação e um novo status para Doliocarpus littoralis (Kubitzki) Fraga \& Stehmann, baseado em uma subespécie de Doliocarpus major J.F.Gmel, e comentada a ocorrência de Neodillenia peruviana Aymard, um novo registro para a flora do Brasil.
\end{abstract}

Palavras-chave: Davilla, Doliocarpus, Neodillenia, Brasil.

\begin{abstract}
Updates on Dilleniaceae from the Brazilian flora are presented from bibliographic databases and herbaria collections. A new species Davilla lanosa Fraga \& Stehmann is described morphologically and illustrated and its affinities to other species of the genus are discussed. The new species is known from two localities in Rondônia and one in southwestern Amazonas, Brazil. A new combination and new status for Doliocarpus littoralis (Kubitzki) Fraga \& Stehmann is also proposed that assumes a specific level to be segregated from Doliocarpus major J. F. Gmel. The occurrence of Neodillenia peruviana Aymar is reported and commented on for Brazil.
\end{abstract}

Key words: Davilla, Doliocarpus, Neodillenia, Brazil.

\section{Introdução}

Os gêneros de Dilleniaceae ocorrentes no Neotrópico pertencem a duas subfamílias distintas. Delimoideae é constituída apenas por Tetracera $L$. que pertence a uma linhagem basal e representa o grupo irmão de todas as demais subfamílias. Os demais gêneros Neotropicais (Curatella L., Davilla Vand., Doliocarpus Rol., Neodillenia Aymard e Pinzona Mart. \& Zucc.) pertencem à subfamília Doliocarpoideae e formam um grupo monofilético irmão das subfamílias Paleotropicais, Dillenioideae e Hibbertioideae (Horn 2009).

A revisão mais recente para Dilleniaceae ocorrentes no Neotrópico foi realizada por Kubitzki (1970, 1971), onde foram reconhecidas ao todo 55 espécies, sendo oito novas para a ciência. Kubitzki (1970) realizou o tratamento taxonômico para 44 espécies de Tetracera, 15 da Australásia, 14 africanas e 15 neotropicais, dentre essas nove foram indicadas para o Brasil. Em seguida, Kubitzki (1971) revisou Davilla, Doliocarpus e os dois gêneros monoespecíficos, Curatella e Pinzona, ambos ocorrentes no Brasil. Em Davilla foram tratadas 18 espécies, com 17 indicadas para o Brasil, e em Doliocarpus, 26 espécies, com 19 para o Brasil, totalizando 47 espécies de Dilleniaceae ocorrendo em território brasileiro.

A partir da análise dos binômios publicados para a família, além da consulta a diversos herbários para a confecção da Lista de Espécies da Flora do Brasil e da revisão taxonômica e filogenia de Davilla, foi possível avaliar o número total de espécies de Dilleniaceae ocorrentes no Brasil, descrever uma nova espécie de Davilla para Rondônia, estabelecer 
uma nova combinação e novo status em Doliocarpus, além de ampliar a distribuição geográfica conhecida de Neodillenia para o território brasileiro.

\section{Material e Métodos}

Foram revisadas as coleções depositadas nos herbários BR, IAN, INPA, MG, NY, R, RB e US. Na descrição morfológica, os caracteres qualitativos e quantitativos foram baseados exclusivamente no material examinado. Na citação do material examinado de Doliocarpus littoralis acrescentou-se o nome do Rio de Janeiro para o espécime Sucre et al. 10054, indicando o atual município que possuía a antiga denominação de Cidade da Guanabara.

\section{Resultados e Discussão}

Após os trabalhos de Kubistzki (1970, 1971) de revisão das Dilleniaceae Neotropicais ocorreu uma ampliação considerável no número de espécies conhecidas tanto para o Neotrópico quanto para o Brasil. Essa ampliação está relacionada, principalmente, a artigos de descrição de novas espécies (Kubitzki 1973, 1980, 1981; Aymard 1992, 1993, 1994, 1996, 1997a,b, 1998a,b, 2002a,b, 2003, 2007a,b; Aymard \& Miller 1994; Fraga \& Aymard 2007; Fraga 2008).

Atualmente são reconhecidas 102 espécies de Dilleniaceae para o Neotrópico, pertencentes aos gêneros Curatella (1), Davilla (31), Doliocarpus (49), Neodillenia (3), Pinzona (1) e Tetracera (17).
No Brasil, os mesmo gêneros se fazem presentes, sendo 30 espécies de Davilla, com apenas D. steyermarkii Kubitzki fora dos limites do país, 34 de Doliocarpus, 15 de Tetracera e uma Neodillenia, além dos dois gêneros monoespecíficos Curatella e Pinzona, totalizando 82 espécies de Dilleniaceae ocorrentes no país (Tab. 1).

No Brasil, a maior riqueza de espécies de Dilleniaceae está na Mata Atlântica (36), seguida da região Amazônica (31) e do Cerrado (14), que sempre foi reconhecido como a região fitogeográfica típica para família, o que está relacionado à abundância e não à riqueza de espécies (Fraga 2009).

Davilla lanosa Fraga \& Stehmann, sp. nov. Tipo: BRASIL. RONDÔNIA: Nova-Mamoré, BR-425, Linha 1, Sítio do Sr. João Presidente, Floresta de terra firme, 28.IX.1996, fr., L.C.B. Lobato, S.S. Almeida, A. Sergio, C. Rosário et A. Gomes 1446 (holótipo MG!; isótipos NY!, RB!). Fig. 1 a-j

Differt ab omnibus species sectionis Davillorum foliis sessilibus, lanceolatis vel spatulatis, margine revoluto, lamina supra tomentosum obsitis, venis pilis sericantibus obsitis, pedicello $1-1,5 \mathrm{~cm}$ longo, staminibus 140-160, seminibus per capsulam 2.

Liana, caule glabro, estriado, castanho quando maduros ou recobertos por tricomas ferrugíneos, seríceos quando jovens. Folhas 7-22 $\times 1,5-8,5 \mathrm{~cm}$, alternas; lâmina subséssil, cartácea, espatulada, oblanceolada ou elíptica, base atenuada,

Tabela 1 - Número de espécies de Dilleniaceae para a região Neotropical e Brasil.

Table 1 - Update of the species number of Dilleniaceae for the Neotropics and Brazil.

\begin{tabular}{lcccl}
\hline Gêneros & \multicolumn{3}{c}{ Número de espécies } & \multicolumn{1}{c}{ Referências * } \\
& Total & Neotrópico & Brasil & \\
\hline Curatella & 1 & 1 & 1 & 2 \\
Davilla & 31 & 31 & 30 & $2,3,4,12,15,16,18,21,23$ \\
Doliocarpus & 49 & 49 & 34 & $2,3,5,6,7,8,11,13,14,17,19,22,23$ \\
Neodillenia & 3 & 3 & 1 & 10,23 \\
Pinzona & 1 & 1 & 1 & 2 \\
Tetracera & 47 & 17 & 15 & $1,3,9,17,20$ \\
Total & & 102 & 82 & \\
\hline
\end{tabular}

(*Referências / References: 1. Kubitzki (1970), 2. Kubitzki (1971), 3. Kubitzki (1973), 4. Kubitzki (1980), 5. Kubitzki (1981), 6. Aymard (1992), 7. Aymard (1993), 8. Aymard \& Miller (1994), 9. Aymard (1996), 10. Aymard (1997a), 11. Aymard (1997b), 12. Aymard (1998a), 13. Aymard (1998b), 14. Ribeiro et al. (1999), 15. Aymard (2002a), 16. Aymard (2002b), 17. Aymard (2003), 18. Aymard (2007a), 19. Aymard (2007b), 20. Fraga \& Aymard (2007), 21. Fraga (2008), 22. Aymard (2008), 23. Fraga \& Stehmann (no presente estudo)). 
ápice agudo ou apiculado, margem inteira, levemente revoluta, verde; face adaxial verrucosa, glabra a esparsamente pilosa na base, face abaxial lanosa; nervura principal canaliculada e impressa na face adaxial, proeminente na face abaxial, serícea em ambas as faces, nervuras secundárias 12-26, convergentes, unindo-se próximo da margem, impressas e glabras na face adaxial, proeminentes e seríceas na face abaxial, nervuras terciárias reticuladas, evidentes em ambas as faces, glabras na face adaxial e lanosas na face abaxial. Inflorescência 7-10 cm compr., simples ou ramificada, axilar ou terminal, 3-25 flores, raque recoberta por tricomas simples, ferrugíneos. Flores com pedicelos 1-1,5 cm compr., recobertos por tricomas simples, ferrugíneos; brácteas basais caducas; 5 sépalas desiguais em tamanho, castanhas quando secas, 3 sépalas externas menores, 0,5-1 cm diâm. quando maduras, desiguais, orbiculares, recobertas por tricomas esparsos externamente, glabras internamente, margens ciliadas, 2 sépalas internas maiores, 1,5-2 cm diâm. quando maduras, orbiculares, externamente rugosas, recobertas por tricomas simples, exparsos, internamente glabras, margens ciliadas, a mais externa sobreposta sobre a mais interna de margem reflexa ambas acrescentes no fruto; pétalas não vistas; estames 140-160, dispostos em círculo em torno dos carpelos, filetes, 4,5-6 mm compr., clavados, glabros, anteras 1-1,5 mm compr., basifixas, oblongas, apiculadas no ápice, glabras, deiscência longitudinal; ovários 2, dialicarpelares. Frutos com 2 sementes por cápsula, 1,8-9 $\times 6-9 \mathrm{~mm}$, quadrangulares a oblongos, estilete persistente, 6,5$8 \mathrm{~mm}$ compr., ereto a sinuoso, glabro, estigma capitado; sementes 6-7 × 5-6 mm, assimétricas, rugosas, glabras, negras, recobertas por arilo papiráceo, branco.

O epíteto específico refere-se à superfície lanosa na face abaxial da folha, caráter presente apenas na nova espécie e em Davilla sellowiana Eichl. e D. kunthii A. St.-Hil. A nova espécie é conhecida de duas localidades em Rondônia e uma ao sul do Amazonas, Brasil, ambas em região de floresta de terra firme na Amazônia.

Davilla lanosa pertence a Sect. Davilla, por apresentar a margem da sépala mais interna reflexa e a margem da sépala adjacente sobreposta, estando ambas acrescentes no fruto, juntamente com 11 espécies divididas em dois grupos morfologicamente distintos. O primeiro grupo (D. kunthii, D. neei Aymard, D. nitida (Vahl.) Kubitzki, D. rugosa Poir. e D. lacunosa Mart.) apresenta espécies 1-ovariadas e normalmente com ampla distribuição geográfica no Neotrópico, com apenas $D$. neei restrita à região oeste da Amazônia brasileira, e D. lacunosa restrita aos cerrados brasileiros. O segundo grupo (D. bahiana Aymard; D. elliptica A. St.-Hil., D. grandiflora A.St.Hil. \& Tul., D. cuspidulata Mart. exEichler, D. glaziovii Eichler and D. cuatrecasasii Aymard) possui espécies 2-ovariadas, com distribuição geográfica restrita a ambientes específicos: $D$. bahiana e $D$. glaziovii são endêmicas de áreas restritas de Mata Atlântica (sul da Bahia e município do Rio de Janeiro, respectivamente), D. elliptica, $D$. grandiflora e D. cuatrecasasii restritas ao Cerrado brasileiro e D. cuspidulata endêmica da Amazônia.

Na Sect. Davilla, a nova espécie é comparada com as espécies do segundo grupo, em função da presença dos dois carpelos livres, com distribuição restrita à floresta de tabuleiros ao sudoseste da Amazônia. Entretanto, D. lanosa difere das espécies deste grupo por apresentar folhas subsésseis (vs. folhas distintamente pecioladas), pedicelo longo, com $1-1,5 \mathrm{~cm}$ compr. (vs. pedicelo curto, com $0,2-0,6 \mathrm{~cm}$ compr.), sépalas internas grandes, de 1,5-2 cm diâm. (vs. sépalas internas pequenas, 0,5-0,7 cm diâm.), estames em número de 140-160 (vs. 60-100) e frutos com duas sementes por cápsula ( $v s$. frutos com uma semente por cápsula).

As características morfológicas aproximam Davilla lanosa das espécies da seção Homalochlaena Kubitzki, que é definida por apresentar sépalas internas não sobrepostas, fortemente comprimidas umas contra as outras. Como essa característica está ausente na nova espécie, não é possível a sua inserção nessa seção, o que demonstra que essas seções podem não formar grupos monofiléticos.

Material examinado: BRASIL. AMAZONAS: Humaitá, Aldeia dos Tenharins, km 120 Rod. Transamazônica, 11.VI.1987, fr., L. Claudio \& J. Luis 76 (INPA). RONDÔNIA: Porto Velho, mata da Universidade Federal de Rondônia, Campus José Ribeiro Filho, 19.IX.2003, fl., F.C.L. Sobreira 2 (INPA).

Doliocarpus littoralis (Kubitzki) Fraga \& Stehmann, comb. et stat. nov. Doliocarpus major J.F. Gmel. subsp. littoralis Kubitzki, Mitt. Bot. Staatssamml. München 9: 65. 1971. Tipo: BRASIL. RIO DE JANEIRO: Cabo Frio. Ponta Gabriel, 22056'48'S, 4201'30'W, 17.IV.1952, fl., L.B. Smith, A. Magnanini, Z.L. Silva, S.L.O. Silva, L. Dau T. Ormond 6664 (holótipo NY!; isótipo US!).

Doliocarpus littoralis é estabelecido a partir de uma subespécie de $D$. major e difere por características morfológicas bem distintas: caule cilíndrico ( $v s$. anguloso) e folhas crasso-coriáceas, 


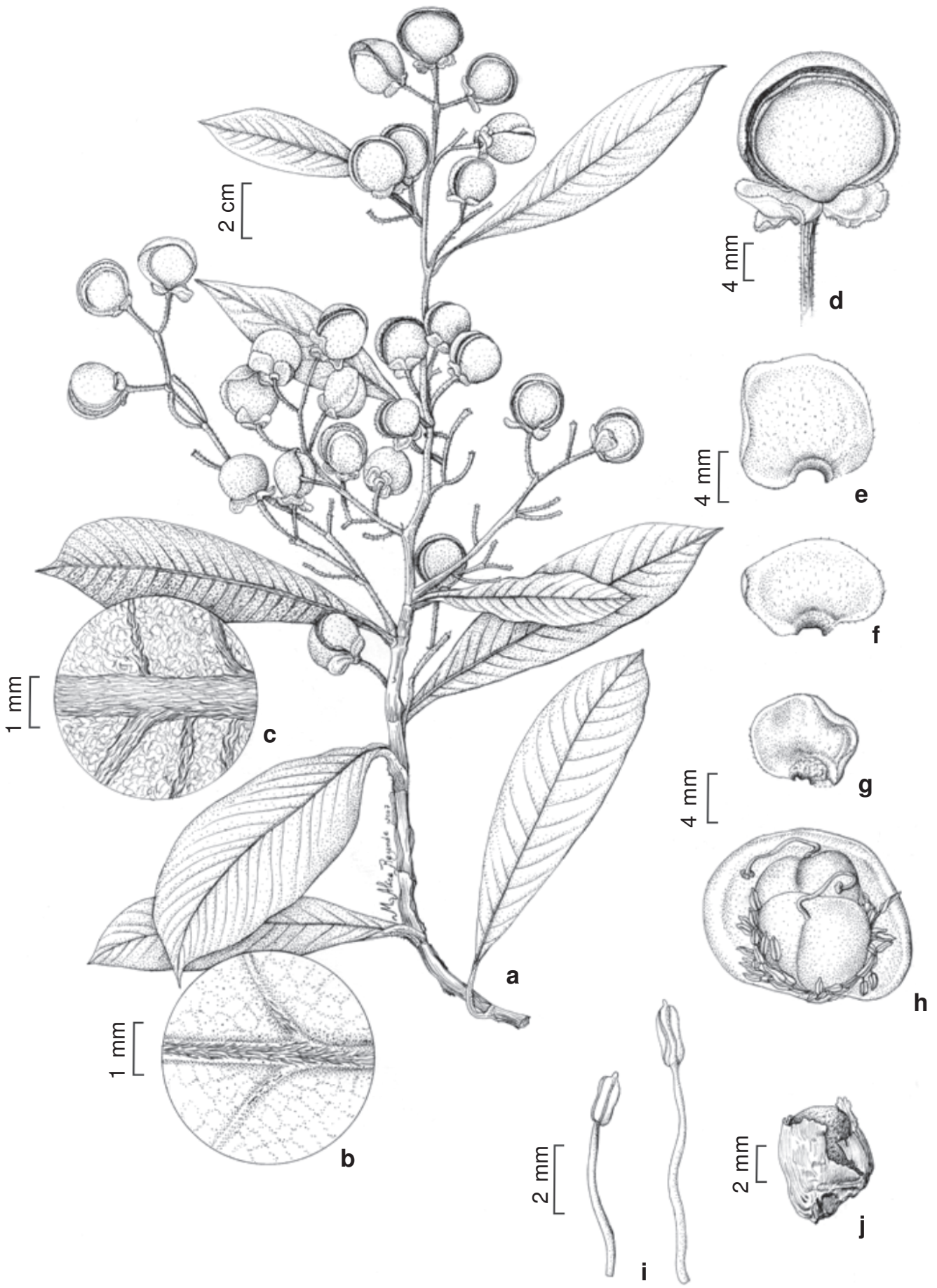

Figura 1 - Davilla lanosa Fraga \& Stehmann - a. ramo florífero; b. detalhe da folha, face adaxial; c. detalhe da folha, face abaxial. d. sépala; e-g. sépalas externas; h. sépala interna, estames e ovários em vista frontal, com uma sépala interna removida; i. estames; j. semente (Lobato et al. 1446).

Figure 1 - Davilla lanosa Fraga \& Stehmann - a. flowering branch; b. detail of the adaxial leaf surface. c. detail of the abaxial leaf surface; d. sepal; e-g. external sepals; h. inner sepal, stamens and ovary in frontal view with one internal sepal removed; i. stamens. j. seed (Lobato et al. 1446). 
glabras, obovadas e com base cuneada ( $v s$. folhas membranáceas, híspido-pubérulas, elípticolanceoladas e com base arredondada). Além disso, D. littoralis possui distribuição restrita ao sul do Espírito Santo e litoral norte do Rio de Janeiro, em floresta de encosta na Mata Atlântica e em áreas de restinga. Desse modo, $D$. littoralis é elevado à categoria de espécie no presente estudo.

Material adicional: BRASIL. RIO DE JANEIRO: Cabo Frio. Along street to town at junction with road to Arraial do Cabo, 17.III.1964, fr., K. Lems s.n. (NY). Arraial do Cabo. Pontal beach, 10.VIII.1953, fl., F. Segadas-Vianna et al. 1350 (NY, R, RB); 27.IX.1953, fr., F. Segadas-Vianna et al. 615 (R, BR). Rio de Janeiro. Morro Queimado, 26.VII.1973, fl., D. Sucre et al. 10054 (RB). ESPÍRITO SANTO: Vila Velha, restinga da Lagoa do Milho, 20.VII.2973, fl., D.S.D. Araujo 327 A.L. Peixoto 197 (RB).

Neodillenia peruviana Aymard, Harvard Pap. Bot. 10: 126. 1997. Tipo: PERU.LORETO: Maynas Province, Las Amazonas, Rio Sucusarí, bosques primários 0320'S, 7255'W, 116 m, 6.XI.1989, fl., R. Vásquez N. Jaramillo 13057 (holótipo MO; isótipos AMAZ, PORT, USM).

Neodillenia peruviana foi descrita por Aymard (1997a), juntamente com outras duas espécies, quando da descrição do gênero Neodillenia, sendo as três ocorrentes na região amazônica extra-brasileira (Colômbia, Equador, Peru, Venezuela). Embora $N$. peruviana tenha sido descrita apenas em 1997, essa espécie já havia sido coletada por Ducke, em 1932, e Froés, em 1949, ambas em São Paulo de Olivença, no estado do Amazonas. Desse modo, permaneceram indeterminadas por cerca de 60 anos e representam os únicos registros conhecidos da espécie para a flora brasileira até os dias de hoje.

Neodillenia foi comparado com Dillenia L. (Dillenioideae) por apresentar flores globosas, estames formando um anel ao redor dos carpelos e anteras com conectivos lineares. Por outro lado o novo gênero foi também comparado com Davilla, Doliocarpus e Pinzona (Doliocarpoideae) por apresentar câmbio sucessivo, inflorescência ramiflora e estiletes longos e peltados, diferenciando Neodillenia desses gêneros pela presença de óvulos ortótropos.

De acordo com Horn (2007) as comparações feitas de Neodillenia com Dillenia, por Aymard (1997a) são equivocadas, pois os estames são totalmente livres, não formam um anel ao redor dos carpelos e os óvulos são campilótropos. Dessa forma Neodillenia apresenta maior afinidade com os gêneros do clado neotropical exclusivo da subfamília Doliocarpoideae (Horn 2009).

Material adicional: BRASIL. AMAZONAS: São Paulo de Olivença, 20.II.1932, fl., A. Ducke s.n. (PORT, RB 25077); Rio Solimões, 4.II.1949, fl., R.L. Froés 24069 (IAN).

\section{Agradecimentos}

Agradecemos os curadores dos herbários Carlos Henrique Franciscon (INPA), Barbara M. Thiers (NY), Luci Senna-Vale (R), Piet Stoffelen (BR), Rafaela Campostrini Forzza(RB), Regina Célia Viana Martins da Silva(IAN), Ricardo de Souza Secco (MG) e Rusty Russell (US) por possibilitarem o acesso às coleções analisadas, especialmente aos materiaistipo; à Maria Alice de Rezende a ilustração da nova espécie; e à Mariana Machado Saavedra o auxílio na diagnose latina e comentários no manuscrito.

\section{Referências}

Aymard, G. 1992. Dilleniaceae Novae Neotropicae. I. Dos Nuevas especies y una nueva subespecie de Doliocarpus de La Guyana Venezoelana. Anales Jardín Botânico de Madrid 49: 195-200.

Aymard, G. 1993. Dilleniaceae Novae Neotropicarum. II. Tres Nuevas especies y dos nuevas subspecies del género Doliocarpus (Dilleniaceae). Novon 3: 317-320.

Aymard, G. 1996. Dilleniaceae Novae Neotropicae: VI. A new species of Tetracera from Panama. Novon 6: 325-327.

Aymard, G. 1997a. Dilleniaceae Novae Neotropicae IX. Neodillenia a new genus from Amazon basin. Harvard Papers in Botany 10: 121-131.

Aymard, G. 1997b. Dilleniaceae Novae Neotropicae, V. El gênero Doliocarpus en Colômbia. Anales Jardín Botânico de Madrid 55: 17-30.

Aymard, G. 1998a. Dilleniaceae novae neotropicae VIII. Two new species of Davilla from Brazil. Brittonia 50: 51-55.

Aymard, G. 1998b. Four new species of Dolicarpus (Dilleniaceae) from Brazilian Amazonian region. Kew Bulletin 53: 713-721.

Aymard, G. 2002a. Davilla papyracea (Dilleniaceae), a new species from Brazil. Kew Bulletin 57: 487-490.

Aymard, G. 2002b. A new species of Davilla (Dilleniaceae) amongst the Flora of São Paulo, Brazil. Acta Botánica Venezuelica 25: 153-159.

Aymard, G. 2003. A new species of Doliocarpus and a new species of Tetracera (Dilleniaceae) from Brazil. Novon 13: 1-4.

Aymard, G. 2007a. Three new species of Davilla (Dilleniaceae) from Brazil. Novon 17: 282-287.

Aymard, G. 2007b. Two new species of Doliocarpus (Dilleniaceae) from Colombia. Novon 17: 288-293. 
Aymard, G. 2008. Dilleniaceae. In: Daly, D.C.; Silveira, M. (orgs.). Primeiro catálogo da flora do Acre, Brasil. EDUFAC, Rio Branco. Pp. 186-187.

Aymard, G. \& Miller, J. 1994. Dilleniaceae Novae Neotropicae. III. Sinopsis y adiciones a las Dilleniaceae del Perú. Candollea 49: 169-182.

Fraga, C.N. 2008. Three new species of Davilla (Dilleniaceae) from Bahia, Brazil. Brittonia 60: 355-361.

Fraga, C.N. 2009. Dilleniaceae. In: Giulietti, A.M.; Rapini, A.; Andrade, M. J. G.; Queiroz, L.P. \& Silva, M.J.C. (orgs.). Plantas raras do Brasil. Conservação Internacional, Belo Horizonte. Pp. 159-160.

Fraga, C.N. \& Aymard, G. 2007. Tetracera forzzae (Dilleniaceae), uma nova espécie para a Zona da Mata de Minas Gerais, Brasil. Novon 17: 433-436.

Horn, J.W. 2007. Dilleniaceae. In: Kubitzki, K. (ed.). The families and genera of vascular plants. Vol. 9. Flowering plants Eudicots: Berberidopsidales et al. Springer, Berlin. Pp. 132-154.

Horn, J.W. 2009. Phylogenetics of Dilleniaceae using sequence data from four plastid loci ( $r b c l$, infa, $r p s 4$, rpl16 INTRON). International Journal of Plant Sciences 170: 794-813.
Kubitzki, K. 1970. Die Gattung Tetracera (Dilleniaceae). Mitteilungen der Botanischen Staatssammlung München 9: 1-98.

Kubitzki, K. 1971. Doliocarpus, Davilla, und verwandte Gattungen (Dilleniaceae). Mitteilungen der Botanischen Staatssammlung München 9: 1-105.

Kubitzki, K. 1973. Neue und bemerkenswerte Neotropische Dilleniaceen. Mitteilungen der Botanischen Staatssammlung München 9: 707-720.

Kubitzki, K. 1980. Eine neue Davilla - arten aus Venezuela. Mitteilungen der Botanischen Staatssammlung München 16: 501-502.

Kubitzki, K. 1981. Zwei neue Doliocarpus - arten aus Venezuela. Mitteilungen der Botanischen Staatssammlung München 17: 237-238.

Ribeiro, J.E.L.S.; Hopkins, M.J.G.; Vicentini, A.; Sthers, C.A.; Costa, M.A.S.; Brito, J.M.; Souza, M.A.D.; Martins, L.H.P.; Lohmana, L.G.; Assunção, P.A.C.L.; Pereira, E.C.; Silva, C.F.; Mesquita, L.C. \& Procópio, L.C. 1999. Flora da Reserva Ducke: Guia de identificação das plantas vasculares de uma floresta de terra-firme na Amazônia Central. INPA, Manaus. 816p. 